\title{
Avaliação da rasagilina no tratamento da Doença de Parkinson: revisão sistemática
}

\author{
Evaluation of rasagiline in the treatment of Parkinson's \\ Disease: systematic review
}

\author{
Evaluación de la rasagiline en el tratamiento de la \\ Enfermedad de Parkinson: revisión sistemática
}

\author{
Luiz Guilherme de Lara1, Taphine Kathleen Szajda², \\ Adriana de Oliveira Christoff ${ }^{3}$
}

\begin{abstract}
1.Bacharel em Farmácia pelo Centro Universitário Autônomo do Brasil (Unibrasil), Escola de Saúde, Curitiba-PR, Brasil. Orcid: https://orcid.org/0000-0002-8744-8590

2.Bacharel em Farmácia pelo Centro Universitário Autônomo do Brasil, Escola de Saúde, Curitiba-PR, Brasil. Orcid: https://orcid.org/0000-0002-0016-6540

3.Doutora em Farmacologia pela Universidade Federal do Paraná. Assessoria do programa de extensão do Centro Universitário Autônomo do Brasil (Unibrasil). Curitiba-PR, Brasil. Orcid: https://orcid.org/00000002-7092-0174
\end{abstract}

\begin{abstract}
Resumo
Introdução. A rasagilina recentemente começou a ser utilizada no Brasil para tratar pacientes com Doença de Parkinson (DP) que apresentam complicações motoras consequentes ao uso da levodopa. Objetivo. Por ser um fármaco novo, o objetivo desta revisão sistemática foi avaliar os efeitos terapêuticos e a segurança da rasagilina utilizada isoladamente e associada a levodopa no tratamento dos sintomas motores de pacientes com DP. Método. As plataformas BVS, EBSCO, PUBMED e SCIELO foram consultadas utilizando os descritores doença de parkinson, rasagilina e levodopa, e seus correspondentes em inglês e espanhol. Após encontrar os artigos publicados entre janeiro de 2010 e agosto de 2020, e filtrar os estudos clínicos randomizados, a leitura dos títulos resultou na seleção daqueles que continham os termos rasagilina e DP. Destes, foram lidos os resumos e selecionados os estudos em que a rasagilina foi combinada levodopa ou não. Resultado. No total, 6 estudos foram selecionados e lidos integralmente. Eles avaliaram a rasagilina combinada a levodopa nos diferentes estágios da doença e concluíram que a associação melhorou os sintomas motores da doença. Um estudo também constatou que, nos primeiros estágios da doença, a monoterapia com rasagilina apresentou efeito terapêutico. A rasagilina, comparada a selegilina, demonstrou eficácia similar no controle motor. Em relação à segurança, três estudos concluíram que a rasagilina é segura devido à baixa ocorrência de efeitos adversos em relação ao grupo que recebeu placebo. Conclusão. A rasagilina é segura e eficaz na redução dos sintomas motores da DP isolada e associada a levodopa.
\end{abstract}

Unitermos. Doença de Parkinson; Rasagilina; Levodopa

\begin{abstract}
Introduction. Rasagiline has recently begun to be used in Brazil to treat patients with Parkinson's Disease (PD) who present motor complications resulting from the use of levodopa. Objective. Because it is a new drug, the objective of this systematic review was to evaluate the therapeutic effects and safety of rasagiline used alone and associated with levodopa in the treatment of the motor symptoms of patients with PD. Method. The BVS, EBSCO, PUBMED and SCIELO platforms were consulted using the descriptors parkinson's disease, rasagiline and levodopa, and their correspondents in English and Spanish. After finding the articles published from January 2010 to August 2020, and filtering the randomized clinical trials, the reading of the titles resulted in the selection of those containing the terms rasagiline and parkinson's disease. From these, abstracts were read and studies in which rasagiline was combined levodopa or not were selected. Results. In total, 6 studies were selected and read integrally. They evaluated the combined levodopa and rasagiline in the different stages of the disease
\end{abstract}


and concluded that the association improved the motor symptoms of the disease. One study also found that in the early stages of the disease, monotherapy with rasagiline had a therapeutic effect. The rasagiline, compared to selegiline, showed similar effectiveness in motor control. In relation to safety, three studies concluded that rasagiline is safe due to the low occurrence of adverse effects in relation to the placebo group. Conclusion. Rasagiline is safe and effective in reducing motor symptoms of isolated and levodopa-associated PD.

Keywords. Parkinson's Disease; Rasagiline; Levodopa

\section{Resumen}

Introducción. La rasagiline ha comenzado a utilizarse recientemente en el Brasil para tratar a los pacientes con Enfermedad de Parkinson (EP) que presentan complicaciones motrices derivadas del uso de la levodopa. Objetivo. Como nuevo fármaco, el objetivo de esta revisión sistemática fue evaluar los efectos terapéuticos y la seguridad de la rasagiline utilizada sola y asociada con la levodopa en el tratamiento de los síntomas motores de los pacientes con EP. Método. Las plataformas BVS, EBSCO, PUBMED y SCIELO fueron consultadas utilizando los descriptores enfermedad de parkinson, rasagiline y levodopa, y sus corresponsales en inglés y español. Después de encontrar los artículos publicados entre enero de 2010 y agosto de 2020, y de filtrar los ensayos clínicos aleatorios, la lectura de los títulos dio lugar a la selección de los que contenían los términos rasagiline y EP. A partir de ellos se leyeron resúmenes y se seleccionaron estudios en los que se combinó o no la rasagiline con levodopa. Resultados. En total, se seleccionaron 6 estudios y se leyeron en su totalidad. Evaluaron la combinación de levodopa y rasagiline en diferentes etapas de la enfermedad y llegaron a la conclusión de que la asociación mejoraba los síntomas motores de la enfermedad. Un estudio también encontró que en las primeras etapas de la enfermedad, la monoterapia con rasagiline tenía un efecto terapéutico. La rasagiline, comparada con la selegiline, mostró una eficacia similar en el control motor. Con respecto a la seguridad, tres estudios concluyeron que la rasagiline es segura debido a la baja incidencia de efectos adversos en relación con el grupo de placebo. Conclusión. La rasagiline es segura y eficaz para reducir los síntomas motores de la EP aislada y asociada a la levodopa.

Palabras clave. Enfermedad de Parkinson; Rasagiline; Levodopa

Trabalho realizado no Centro Universitário Autônomo do Brasil (Unibrasil), Curitiba-PR, Brasil.

\section{INTRODUÇÃO}

O mesilato de rasagilina, fármaco antiparkinsoniano, foi aprovado pela Agência Nacional de Vigilância Sanitária (ANVISA), para ser utilizado no Brasil, por meio da publicação do parecer público no dia 01 de junho de $2015^{1}$. Em 2017, o medicamento foi incorporada ao SUS (Sistema Único de Saúde) pelo Ministério da Saúde, Secretaria da Ciência, Tecnologia e Insumos Estratégicos, através da Portaria No 27, de 02 de agosto de 2017, para ser utilizado em combinação com a levodopa para o tratamento dos 
pacientes com doença de Parkinson que apresentam complicações motoras ${ }^{2}$. Ainda em 2017, a CONITEC (Comissão Nacional de Incorporação de Tecnologias no SUS), após análise de evidência clínica, avaliação econômica e análise de impacto orçamentário, considerou que o mesilato de rasagilina é eficaz e seguro no tratamento dos pacientes que utilizam a levodopa e apresentam complicações motoras e posicionou-se favorável à incorporação do fármaco ao SUS ${ }^{3}$. Em seguida, a Secretaria de Atenção à Saúde aprovou o Protocolo Clínico e Diretrizes Terapêuticas da Doença de Parkinson através da Portaria Conjunta No 10, de 31 de Outubro de 2017, incluindo a rasagilina entre as opções farmacológicas para 0 tratamento ${ }^{4}$.

A Doença de Parkinson (DP) é uma doença neurodegenerativa caracterizada pela morte dos neurônios dopaminérgicos da substância nigra ${ }^{5}$. Essa alteração se deve a uma condição conhecida como "degeneração neuronal", que é responsável pela redução da quantidade de dopamina produzida e liberada em regiões anatômicas bem definidas do SNC, especialmente na região nigroestriatal, o que resulta numa transmissão nervosa deficiente e leva ao surgimento dos sinais e sintomas dessa doença. O diagnóstico da DP depende da presença dos sinais motores como bradicinesia, tremor de repouso e rigidez, e pode ser complementado pela manifestação de alguns sinais não motores como déficits cognitivos, distúrbios do humor, do sono, depressão e incontinência urinária ${ }^{6}$. 
Com o passar do tempo e a evolução da doença, que está relacionada a qualidade de vida dos pacientes, a levodopa, medicamento considerado padrão ouro para o tratamento do parkinson, diminui a sua efetividade, sendo necessários reajustes das doses $^{6}$. Com 0 aumento da neurodegeneração as limitações físicas interferem na mobilidade do paciente e o desempenho funcional das atividades diárias fica deficiente, o que contribui para a piora da qualidade de vida e pode levar ao aumento dos sinais da doença?.

O ajuste de dose dos medicamentos empregados, em especial, da levodopa, um precursor dopaminérgico, é necessário porque, logo quando a DP é diagnosticada, ainda há uma reserva de neurônios dopaminérgicos, a qual vai reduzindo durante a evolução da doença. A rasagilina é um inibidor seletivo e irreversível da isoforma B da enzima monoaminoxidase, resultando na diminuição da degradação da dopamina e elevação da concentração do neurotransmissor em diversas áreas do cérebro ${ }^{8}$.

A associação com outros medicamentos é de vital importância porque o ajuste das doses das medicações é limitado pela toxicidade e pelos efeitos colaterais, sendo necessários abordagens diferentes para conseguir atingir o objetivo principal do tratamento, sendo esse a redução dos sinais e sintomas através do aumento da dopamina. Os efeitos adversos e colaterais, o número de medicações e os horários múltiplos de administração por dia, têm influência 
de forma direta na adesão do paciente ao tratamento medicamentoso ${ }^{9}$.

Dessa forma, o acompanhamento farmacoterapêutico é uma ação primordial em pacientes que fazem uso de medicação contínua. Quando utilizados de forma incorreta, os medicamentos podem trazer problemas que podem interferir no tratamento do paciente, como: dificuldade de adesão ao tratamento farmacológico, presença de reações adversas e interações medicamentosas ${ }^{10}$.

No final de 2019, o Ministério da Saúde publicou uma nova versão da Relação Nacional de Medicamentos Essenciais (RENAME) e, entre os medicamentos incluídos, a rasagilina foi inserida na Relação Nacional de Medicamentos do Componente Especializado da Assistência Farmacêutica. Nesta lista estão os medicamentos para pacientes com quadros clínicos crônicos que possuem custos de tratamento mais elevados ou mais complexos ${ }^{11}$. De acordo com a Secretaria de Estado da Saúde do Paraná, a rasagilina é classificada como pertencente ao grupo $1 \mathrm{~A}^{12}$ e a responsabilidade de aquisição do medicamento é centralizada no Ministério da Saúde que fornece o produto às Secretarias de Saúde dos Estados e do Distrito Federal ${ }^{11}$.

Em se tratando de um fármaco novo no Brasil é necessário que se busque na literatura dados sobre os efeitos terapêuticos e a segurança da rasagilina. Por isso, o objetivo do presente trabalho é realizar uma revisão sistemática da literatura para avaliar a segurança e os efeitos terapêuticos da rasagilina utilizada isoladamente e associada a levodopa 
no tratamento dos sintomas motores de pacientes diagnosticados com a DP.

\section{MÉTODO}

Este estudo consistiu em uma revisão sistemática da literatura. Foram realizadas buscas de artigos nas bases de dados EBSCO, BVS (biblioteca virtual em saúde), PUBMED e SCIELO, utilizando os descritores: doença de parkinson, rasagilina, levodopa, e os seus correspondentes em inglês e espanhol.

Os critérios de inclusão considerados foram os artigos escritos em português, inglês ou espanhol, publicados entre 01 de janeiro de 2010 a 17 de agosto de 2020, de estudos clínicos controlados randomizados (RCT) realizados em pacientes com a DP que fizeram o uso da rasagilina associada a levodopa, assim como os estudos que utilizaram a rasagilina de forma isolada. Alguns artigos não compreendidos no período estipulado foram trazidos na discussão para complementar algumas ideias como, por exemplo, os estudos PRESTO (2005) ${ }^{13}$ e TEMPO (2002) ${ }^{14}$, ambos publicados pela Parkinson Study Group, foram os estudos iniciais que avaliaram a rasagilina e corroboram com os resultados desta revisão.

Os critérios de exclusão considerados foram os artigos incompletos, os estudos de metanálise, teses e as revisões sistemáticas e qualitativas. Também foram excluídos os protocolos de pesquisa clínica que não apresentaram resultados (clinical trial). 
A busca dos artigos começou com a utilização do descritor doença de parkinson. Em seguida, foi adicionado o descritor rasagilina e o operador booleano AND. Após, foi incluído o descritor levodopa, também combinada com o operador booleano AND. Dos resultados obtidos, foi aplicado o filtro de período de publicação e, posteriormente, os estudos clínicos randomizados. Então, a leitura dos títulos e resumos foi realizada aos pares, aplicando os critérios de inclusão e exclusão. Para serem considerados, os títulos deveriam conter os termos rasagilina e doença de parkinson. Posteriormente, ocorreu a leitura dos abstracts, que deveriam trazer a informação de que tiveram grupos que receberam somente a rasagilina e grupos em que foi feita a combinação com a levodopa, seguidos da comparação estatística informando se houve diferença significante entre os grupos ou não. Por fim, foi feita a leitura na íntegra dos artigos selecionados que permaneceram dentro dos critérios adotados.

Dos artigos selecionados foram analisados o tempo de tratamento, a melhora dos sintomas motores da doença com a adição da rasagilina, a ocorrência de efeitos adversos e interações medicamentosas.

\section{RESULTADOS}

As buscas dos artigos nas bases de dados aconteceram no mês de agosto de 2020 e foram encontrados 169 artigos. Dos 80 artigos encontrados na BVS, foram excluídos: 3 artigos incompletos, 6 estudos de metanálise, 27 revisões, 
26 artigos que não atenderam aos objetivos do estudo e 16 artigos já haviam sido considerados em outra base da dados. Para os 11 artigos do PUBMED, foram excluídos: 2 revisões, 4 artigos que não atenderam aos objetivos do estudo, 1 artigo encontrado com o descritor em espanhol que já havia sido considerado com o descritor em inglês e 1 estudo já selecionado em outra base de dados. Por fim, dos 78 artigos da EBSCO, 22 foram excluídos pela própria plataforma por duplicação e dos 56 restantes: 3 eram estudos de metanálise, 6 revisões, 41 não atenderam aos objetivos do estudo e 5 já haviam sido considerados em outra base de dados. Portanto, foram selecionados 2 artigos da BVS, 3 do PUBMED e 1 da EBSCO, totalizando 6 estudos.

O processo de busca e seleção está apresentado na Figura 1. Não foi construído fluxograma para o portal SCIELO uma vez que nenhum artigo foi selecionado.

Os seis artigos selecionados estão apresentados na Tabela 1.

\section{DISCUSSÃO}

Esta revisão avaliou a segurança e os efeitos terapêuticos da rasagilina isolada e associada a levodopa no tratamento dos sintomas motores da DP a partir da revisão de seis estudos internacionais devido a não existência de trabalhos nacionais publicados para este assunto. 
Figura 1. Organograma de busca e seleção dos artigos que avaliaram os efeitos da rasagilina isolada ou associada a levodopa nos pacientes com doença de parkinson.

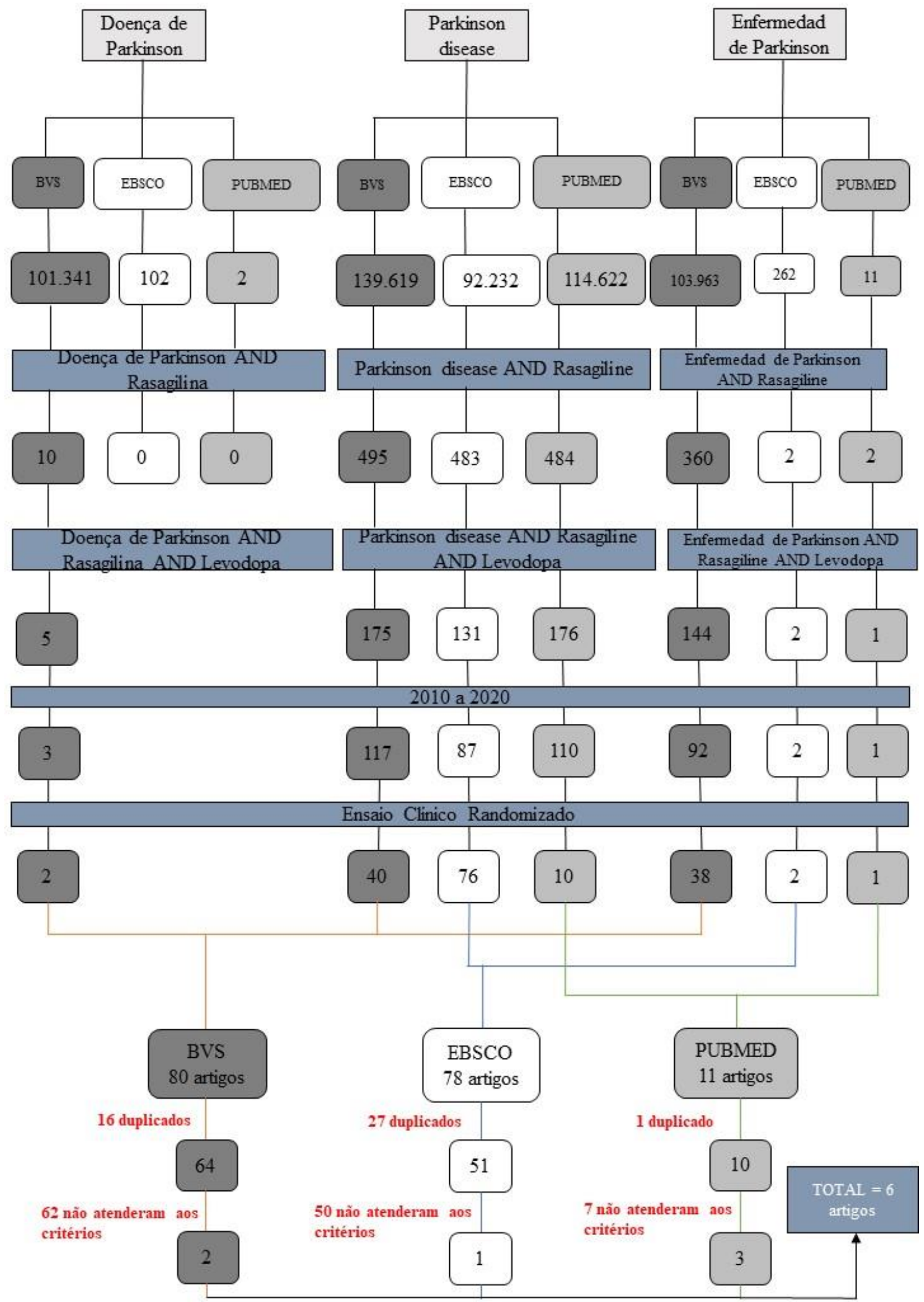


Tabela 1. Apresentação dos 6 artigos incluídos que avaliaram a eficácia e segurança da rasagilina em pacientes com doença de parkinson.

\begin{tabular}{|c|c|c|c|c|}
\hline Título/Autor/Ano & Objetivo & $\begin{array}{c}\text { No de } \\
\text { Participantes/ } \\
\text { Tratamento }\end{array}$ & Efeitos Adversos & Resultado \\
\hline $\begin{array}{l}\text { Effect of rasagiline } \\
\text { as adjunct therapy } \\
\text { to levodopa on } \\
\text { severity of OFF in } \\
\text { Parkinson's disease }\end{array}$ & $\begin{array}{l}\text { Determinar a } \\
\text { segurança, } \\
\text { tolerabilidade } \\
\text { e eficácia da } \\
\text { rasagilina em } \\
\text { pacientes } \\
\text { com } \\
\text { parkinson } \\
\text { tratados com } \\
\text { levodopa e } \\
\text { com } \\
\text { flutuações } \\
\text { motoras. }\end{array}$ & $\begin{array}{l}105 \text { participantes. } \\
\text { Grupos: } \\
32 \text { rasagilina } \\
\text { 1mg/dia + } \\
\text { levodopa; } 36 \\
\text { entacapone } \\
200 \mathrm{mg} / \text { dose de } \\
\text { levodopa; } \\
37 \text { placebo + } \\
\text { levodopa. } \\
\text { Tempo do estudo: } \\
18 \text { semanas. }\end{array}$ & $\begin{array}{l}\text { Não avaliado pelo } \\
\text { estudo. }\end{array}$ & $\begin{array}{l}\text { A rasagilina } \\
\text { combinada a } \\
\text { levodopa é } \\
\text { eficaz na } \\
\text { redução dos } \\
\text { sintomas } \\
\text { motores. } \\
\text { Não houve } \\
\text { diferença } \\
\text { significante } \\
\text { entre os } \\
\text { efeitos da } \\
\text { rasagilina e } \\
\text { entacapone. }\end{array}$ \\
\hline $\begin{array}{l}\text { Rapid onset of } \\
\text { efficacy of } \\
\text { rasagiline in early } \\
\text { Parkinson's disease } \\
\text { Marsala } 2013^{16}\end{array}$ & $\begin{array}{c}\text { Avaliar o } \\
\text { efeito agudo } \\
\text { da rasagilina } \\
\text { nos sintomas } \\
\text { motores em } \\
\text { pacientes } \\
\text { italianos com } \\
\text { doença de } \\
\text { parkinson. }\end{array}$ & $\begin{array}{l}102 \text { participantes. } \\
\text { Grupos: } \\
26 \text { rasagilina } 1 \mathrm{mg} ; \\
76 \text { rasagilina + } \\
\text { levodopa e/ou } \\
\text { agonistas } \\
\text { dopaminérgicos. } \\
\text { Tempo do estudo: } \\
4 \text { semanas. }\end{array}$ & $\begin{array}{l}\text { Não avaliado pelo } \\
\text { estudo. }\end{array}$ & $\begin{array}{l}\text { A rasagilina } \\
\text { teve efeito } \\
\text { terapêutico } \\
\text { em ambos } \\
\text { os grupos e } \\
\text { não houve } \\
\text { diferença } \\
\text { significante } \\
\text { entre eles. }\end{array}$ \\
\hline $\begin{array}{l}\text { Efficacy of } \\
\text { rasagiline and } \\
\text { selegiline in } \\
\text { Parkinson's } \\
\text { disease: a head-to- } \\
\text { head 3-years } \\
\text { retrospective case- } \\
\text { control study } \\
\text { Cereda } 2017^{17}\end{array}$ & $\begin{array}{l}\text { Comparar a } \\
\text { eficácia da } \\
\text { selegilina e } \\
\text { rasagilina no } \\
\text { controle dos } \\
\text { sintomas } \\
\text { motores de } \\
\text { pacientes } \\
\text { italianos com } \\
\text { Parkinson. }\end{array}$ & $\begin{array}{l}340 \text { participantes. } \\
\text { Grupos: } \\
85 \text { levodopa + } \\
\text { selegilina } 5 \text { ou } \\
10 \mathrm{mg} / \text { dia; } \\
85 \text { levodopa + } \\
\text { rasagilina } 0,5 \text { ou } \\
1 \mathrm{mg} / \text { dia. } \\
170 \text { levodopa. } \\
\text { Tempo do estudo: } \\
3 \text { anos. }\end{array}$ & $\begin{array}{l}\text { Não avaliado pelo } \\
\text { estudo. }\end{array}$ & $\begin{array}{l}\text { Selegilina e } \\
\text { rasagilina } \\
\text { têm eficácia } \\
\text { similar no } \\
\text { controle } \\
\text { motor. }\end{array}$ \\
\hline $\begin{array}{l}\text { Efficacy and safety } \\
\text { of rasagiline as an } \\
\text { adjunct to levodopa } \\
\text { treatment in } \\
\text { Chinese patients } \\
\text { with Parkinson's } \\
\text { disease: a } \\
\text { randomized, } \\
\text { double-blind, } \\
\text { parallel-controlled, } \\
\text { multi-centre trial }\end{array}$ & $\begin{array}{l}\text { Avaliar a } \\
\text { segurança e } \\
\text { eficácia da } \\
\text { rasagilina em } \\
\text { pacientes } \\
\text { chineses com } \\
\text { parkinson } \\
\text { tratados com } \\
\text { levodopa e } \\
\text { com } \\
\text { flutuações } \\
\text { motoras. }\end{array}$ & $\begin{array}{l}244 \text { participantes } \\
\text { Grupos: } \\
119 \text { rasagilina } 1 \mathrm{mg}+ \\
\text { levodopa/bense- } \\
\text { razida; } \\
125 \text { placebo } 1 \mathrm{mg}+ \\
\text { levodopa/bense- } \\
\text { razida. } \\
\text { Tempo do estudo: } 12 \\
\text { semanas. }\end{array}$ & $\begin{array}{l}\text { Comuns (>1\%): } \\
\text { Vertigem, dor de } \\
\text { cabeça, dor } \\
\text { muscular, fadiga, } \\
\text { dor, náusea, } \\
\text { xerostomia, } \\
\text { aumento das } \\
\text { enzimas } \\
\text { hepáticas, dor } \\
\text { abdominal, } \\
\text { eritema. } \\
1 \text { hospitalização } \\
\text { por episódio de } \\
\text { doença pulmonar } \\
\text { obstrutiva crônica } \\
\text { no grupo da } \\
\text { rasagilina. } \\
2 \text { participantes do } \\
\text { grupo da rasagilina } \\
\text { tiveram redução do } \\
\text { número } \\
\text { leucócitos. de }\end{array}$ & $\begin{array}{l}\text { O grupo que } \\
\text { recebeu } \\
\text { rasagilina } \\
\text { apresentou } \\
\text { nelhor controle } \\
\text { da doença } \\
\text { medidos } \\
\text { através dos } \\
\text { sintomas } \\
\text { clínicos. A } \\
\text { rasagilina } \\
\text { também } \\
\text { demonstrou } \\
\text { maior } \\
\text { segurança. }\end{array}$ \\
\hline
\end{tabular}


Tabela 1 (cont.). Apresentação dos 6 artigos incluídos que avaliaram a eficácia e segurança da rasagilina em pacientes com doença de parkinson.

\begin{tabular}{|c|c|c|c|c|}
\hline Título/Autor/Ano & Objetivo & $\begin{array}{c}\mathbf{N}^{\circ} \mathrm{de} \\
\text { Participantes/ } \\
\text { Tratamento }\end{array}$ & Efeitos Adversos & Resultado \\
\hline $\begin{array}{l}\text { Adjunct rasagiline to } \\
\text { treat Parkinson's } \\
\text { disease with motos } \\
\text { fluctuations: a } \\
\text { randomized, double- } \\
\text { blind study in China }\end{array}$ & $\begin{array}{c}\text { Avaliar a eficácia } \\
\text { e a segurança da } \\
\text { rasagilina } \\
\text { associada a } \\
\text { levodopa em } \\
\text { pacientes } \\
\text { chineses com } \\
\text { parkinson. }\end{array}$ & $\begin{array}{l}324 \\
165 \text { levodopa + } \\
\text { rasagilina } \\
\text { 1mg/dia; } \\
159 \text { levodopa + } \\
\text { placebo 1mg/dia. } \\
\text { Tempo do } \\
\text { estudo: } 16 \\
\text { semanas. }\end{array}$ & $\begin{array}{l}\text { Comuns ( } \geq 1 \%) \text { : } \\
\text { Discinesia, } \\
\text { agravamento ou } \\
\text { progressão dos } \\
\text { sintomas da } \\
\text { doença, aumento } \\
\text { das enzimas } \\
\text { hepáticas, náusea, } \\
\text { quedas, fadiga, } \\
\text { constipação, } \\
\text { distensão } \\
\text { abdominal, } \\
\text { diarreia, } \\
\text { nasofaringite. }\end{array}$ & $\begin{array}{l}\text { O grupo } \\
\text { tratado com } \\
\text { rasagilina } \\
\text { associada a } \\
\text { levodopa } \\
\text { apresentou } \\
\text { maior eficácia } \\
\text { e segurança } \\
\text { quando } \\
\text { comparada } \\
\text { com o } \\
\text { placebo. }\end{array}$ \\
\hline $\begin{array}{l}\text { Efficacy and safety } \\
\text { of adjunctive } \\
\text { rasagiline in } \\
\text { Japanese } \\
\text { Parkinson's disease } \\
\text { with wearing-off } \\
\text { phenomena: a } \\
\text { phase 2/3, } \\
\text { randomized, double- } \\
\text { blind, placebo- } \\
\text { controled, multi- } \\
\text { centre study } \\
\text { Hattori } 2018^{20}\end{array}$ & $\begin{array}{l}\text { Avaliar a eficácia } \\
\text { e segurança da } \\
\text { rasagilina } \\
\text { associada a } \\
\text { levodopa em } \\
\text { pacientes } \\
\text { japoneses com } \\
\text { parkinson e } \\
\text { fenômeno } \\
\text { wearing-off. }\end{array}$ & $\begin{array}{l}404 \text { participantes } \\
\text { Grupos: } \\
141 \text { levodopa + } \\
\text { placebo; } \\
134 \text { levodopa + } \\
\text { rasagilina } \\
\text { 0,5mg/dia; } \\
129 \text { levodopa + } \\
\text { rasagilina } 1 \mathrm{mg} / \text { dia } \\
\text { Tempo do estudo: } \\
26 \text { semanas. }\end{array}$ & $\begin{array}{l}\text { Comuns: } \\
\text { Nasofaringite, } \\
\text { discinesia, } \\
\text { quedas, } \\
\text { contusão. }\end{array}$ & $\begin{array}{c}\text { Os grupos } \\
\text { que } \\
\text { receberam } \\
\text { rasagilina } \\
\text { apresentaram } \\
\text { respostas } \\
\text { mais eficazes. } \\
\text { Houve } \\
\text { diferença } \\
\text { significante } \\
\text { entre o grupo } \\
\text { que recebeu } \\
\text { 1mg/dia de } \\
\text { rasagilina em } \\
\text { relação ao } \\
\text { placebo }\end{array}$ \\
\hline
\end{tabular}

Marsala $2013^{16}$ constataram que tanto para os pacientes italianos iniciantes na terapia para a DP quanto para os que já faziam uso da levodopa, combinada ou não com agonistas dopaminérgicos, houve efeito terapêutico desde a primeira semana de tratamento com rasagilina. Comparações entre os grupos revelaram que não houve diferença significante entre eles. Como os participantes que receberam apenas rasagilina apresentaram os mesmos resultados de melhora dos sintomas motores sem a 
levodopa, isso confirma que sozinha, nos estágios iniciais da doença caracterizada por sintomas mais amenos, é segura e eficaz. Stocchi $2011^{15}$ também avaliaram a eficácia da rasagilina combinada a levodopa por um período maior e concluíram que a adição da rasagilina melhorou, em relação ao placebo, os sintomas motores dos pacientes nos estágios mais avançados da doença. A rasagilina, a longo prazo, foi eficaz na redução dos sintomas motores da doença em pacientes japoneses ${ }^{21}$.

Todos esses resultados são semelhantes ao do estudo de PRESTO publicado em 2005 pela Parkinson Study Group ${ }^{13}$, no qual o objetivo foi determinar a segurança, eficácia e tolerabilidade da rasagilina associada a levodopa em pacientes com DP e com flutuações motoras. Além de reduzir o sintoma motor, o tratamento com rasagilina possibilitou a redução da dosagem diária de levodopa. No estudo de TEMPO ${ }^{14}$, também publicado pela Parkinson Study Group em 2002, a monoterapia com rasagilina foi eficaz no tratamento de pacientes nos primeiros estágios da doença. Apesar da grande diferença entre o tempo de uso da rasagilina nos estudos de Marsala $2013^{16}$ e Stocchi $2011^{15}$, comparados aos dos estudos PRESTO ${ }^{13}$ e TEMPO $^{14}$, a melhora dos sintomas da doença começaram a ser percebidos em poucas semanas e, provavelmente, os sintomas melhorariam mais com o tempo maior de tratamento. Portanto, a rasagilina é eficaz quando administrada sozinha ou associada com a levodopa se mostrando eficaz nos diferentes estágios da doença e a 
característica multicêntrica dos estudos possibilita presumir que a rasagilina poderá ser eficaz para os pacientes brasileiros.

Ao avaliar a eficácia da rasagilina como terapia adjuvante a levodopa/benserazida em chineses com DP que apresentavam flutuações motoras, Zhang $2013^{18}$ concluíram que os pacientes que fizeram uso da rasagilina tiveram melhora significativa do tremor, rigidez e bradicinesia em relação ao grupo que recebeu placebo. Além disso, foi percebido maior redução dos períodos on e off nesse grupo. Zhang $2018^{19}$ realizaram o mesmo estudo em pacientes chineses com flutuações motoras, sendo que, a diferença entre os estudos foi a intervenção, uma vez que não houve a associação de benserazida ou carbidopa com a levodopa. A diferença entre o grupo que recebeu rasagilina versus placebo foi favorável para o primeiro grupo devido a maior redução dos sintomas motores nos períodos on e off.

Quando a levodopa começou a ser usada cronicamente no tratamento de pacientes parkinsonianos, com o tempo, oscilações ou flutuações motoras eram desenvolvidas ${ }^{22}$. Essas flutuações estavam relacionadas com a concentração plasmática de levodopa ${ }^{23}$. Quando o paciente estava no período on, sob a ação da medicação, os sintomas da doença melhoravam significativamente e no período off, no qual há a redução da concentração plasmática de levodopa, houve piora dos sintomas ${ }^{23}$. No início do tratamento com drogas antiparkinsonianas, como a levodopa, parte da medicação biodisponível no cérebro é convertida em dopamina dentro 
do neurônio dopaminérgico e é estocada em vesículas para ser utilizada posteriormente, e a outra parte é convertida em dopamina e utilizada imediatamente no terminal sináptico. Com a morte progressiva dos neurônios dopaminérgicos, a maior parte da medicação administrada passa a ser convertida em dopamina e é imediatamente utilizada, o que leva a uma redução da duração do efeito do fármaco, caracterizando o período off ${ }^{5}$.

A levodopa é o padrão ouro do tratamento do parkinson por ser precursora da dopamina ${ }^{24}$. Para ser biotransformada a levodopa sofre a ação da enzima DOPA-descarboxilase, que está presente em altas concentrações no sangue periférico e nos tecidos ${ }^{24}$. Como grande parte da levodopa absorvida já é convertida em dopamina, apenas uma pequena concentração consegue atravessar a barreira hematoencefálica e, para aumentar a biodisponibilidade no SNC, é possível adicionar inibidores da DOPA-descarboxilase às doses de levodopa ${ }^{24}$. No entanto, a biodisponibilidade de dopamina na região nigroestriatal é ainda menor em razão da metabolização pelas enzimas MAO-A e MAO-B, sendo que a concentração da isoforma $B$ é maior nessa região do cérebro ${ }^{25}$. A inibição seletiva da MAO-B, realizada pela selegilina ou rasagilina, pode ajudar na melhora dos sintomas da doença de parkinson com a elevação das concentrações de dopamina sem a necessidade de aumentar as doses de levodopa. Os resultados de Zhang $2013^{18} \mathrm{e}$ Zhang $2018^{19}$ permitem concluir que quando há associação da rasagilina com a levodopa, ocorre uma combinação dos 
efeitos terapêuticos das duas medicações e, consequentemente, melhora das complicações motoras resultantes da terapia com levodopa.

No fenômeno wearing-off, que está relacionado com o final de cada dose de levodopa, o paciente apresenta sintomas motores e/ou não motores até a nova administração do medicamento ${ }^{26}$. As primeiras manifestações motoras da ocorrência deste fenômeno são tremores e imobilidade ao acordar que melhoram após a primeira dose diária de levodopa ${ }^{27}$. Já as manifestações não motoras podem ser a ansiedade, fadiga ou depressão ${ }^{28}$. Quando o fenômeno wearing-off está presente há o indício de que a doença está em fase mais complexa ${ }^{29}$.

Hattori $2018^{20}$ avaliaram a eficácia de diferentes dosagens de rasagilina em melhorar o desempenho motor de pacientes japoneses com fenômeno wearing-off e concluíram que, após 26 semanas, a dosagem de $1 \mathrm{mg} /$ dia proporcionou maior redução do período off quando comparada ao placebo. Já a dosagem de 0,5mg/dia não apresentou diferença significante em relação ao placebo. Neste estudo não foram feitas análises estatísticas entre os grupos que receberam doses diferentes de rasagilina mas, ao comparar os resultados desse estudo com os do estudo PRESTO ${ }^{13}$, que investigou a eficácia da rasagilina associada a levodopa em pacientes parkinsonianos com flutuações motoras percebeuse que, embora a dosagem de $0,5 \mathrm{mg} / \mathrm{dia}$ de rasagilina proporcione menores efeitos adversos, a dose de $1 \mathrm{mg} / \mathrm{dia}$ apresentou melhor resposta na redução do período off. A 
semelhança entre os resultados do Hattori $2018^{20}$ e do estudo PRESTO ${ }^{13}$ confirma a eficácia de $1 \mathrm{mg} /$ dia de rasagilina em melhorar os sintomas motores de pacientes tratados com levodopa e com complicações motoras.

No estudo de Zhang $2013^{18}$ foi relatado que tanto no grupo que recebeu o medicamento quanto no grupo placebo tiveram participantes que foram descontinuados do estudo devido à baixa adesão ao tratamento. No artigo não é relatado o motivo, mas é provável que os participantes, em razão de possíveis efeitos colaterais indesejados, tenham diminuído ou cessado a frequência de administração das dosagens por conta própria, o que interferiria nos resultados encontrados pelo estudo. Contudo, como nesse estudo era permitido o uso de outros medicamentos, como bromocriptina, amantadina, piribedil e pramipexol, é possível que interações medicamentosas tenham ocorrido nesses pacientes. Além disso, não é relatado no estudo se os pacientes apresentavam comorbidades que estavam sendo tratadas farmacologicamente ou se eles faziam o uso de fórmulas caseiras para tratá-las.

Ao comparar a eficácia da selegilina e rasagilina quando associadas a levodopa, Cereda $2017^{17}$ concluíram que elas são similares no controle dos sintomas motores. Entretanto, a selegilina, após o processo de biotransformação, gera como metabólitos principais a anfetamina e metanfetamina ${ }^{8}$ que, por serem farmacologicamente ativos, podem provocar efeitos como ansiedade e insônia ${ }^{30}$. As anfetaminas e a metanfetamina podem atuar promovendo maior liberação de 
noradrenalina, serotonina e dopamina agindo em neurônios pré-sinápticos ${ }^{30}$, o que pode sugerir uma melhora dos efeitos parkinsonianos pelo aumento da dopamina, mas complicações como o aparecimento de efeitos colaterais importantes, principalmente devido ao aumento da noradrenalina, provocando os sintomas já relatados incluindo insônia, taquicardia, elevação da pressão arterial, entre outros. No estudo de Cereda $2017^{17}$ não houve diferença entre os pacientes tratados com rasagilina e selegilina, mostrando que esse aumento de dopamina pode não ser significativo a ponto de promover alguma melhora, o que torna o uso da rasagilina mais seguro nesse aspecto.

Como o único metabólito da rasagilina formado durante o metabolismo hepático é o 1-aminoidan ${ }^{8}$, a ansiedade e insônia e demais manifestações não foram reportadas, uma vez que esse metabólito não apresenta afinidade pelos receptores catecolaminérgicos e serotoninérgicos ${ }^{8,31}$. Além desses problemas, os metabólitos da selegilina podem provocar aumento da pressão arterial e deve-se considerar que o paciente com parkinson, normalmente é idoso, e provavelmente apresenta doenças cardiovasculares e o uso da selegilina poderia trazer descompensação da PA, que quase sempre está controlada com agentes antihipertensivos. Sendo assim, a ragasilina é mais indicada.

Em um estudo feito com a combinação da rasagilina com a levodopa mostrou melhora dos distúrbios do sono de pacientes com parkinson ${ }^{32}$. A explicação dada pelos autores 
é que os inibidores da MAO-B aumentam a concentração de melatonina na glândula pineal.

Com relação aos efeitos adversos alguns participantes do estudo de Zhang $2013^{18}$, que receberam rasagilina, apresentaram dor de cabeça, náusea, vertigem, agitação, dor muscular, fadiga, xerostomia e dor abdominal. Zhang $2018^{19}$ perceberam que o grupo de pacientes que recebeu apenas rasagilina apresentou menores quadros de discinesias quando comparado com o grupo que recebeu placebo. Insônia e enzimas hepáticas (AST e ALT) elevadas foram observadas em alguns participantes ${ }^{19}$. Uma paciente não diabética dos Emirados Árabes apresentou hipoglicemia grave 4 dias após a rasagilina ter sido prescrita como monoterapia em substituição à rotigotina, levodopa/carbidopa e entacapone ${ }^{33}$. Como este relato era inédito, não foi possível investigar maiores dados de segurança para tal condição. Porém, há relato de casos de hipoglicemia grave após o uso de algumas classes de fármacos, como quinina, $\beta$-bloqueadores, inibidores da ECA, inibidores seletivos da recaptação de serotonina e inibidores não seletivos da enzima $\mathrm{MAO}^{34}$. Como há o relato da ocorrência de síndrome serotoninérgica em pacientes que utilizaram inibidores não seletivos da $M A O$, especula-se que embora a rasagilina seja inibidora seletiva da MAOB há a possibilidade de uma inibição da MAO-A, o que resultou no aparecimento do efeito serotoninérgico com o aumento da sensibilidade à insulina e maior liberação desta, resultando 
no quadro hipoglicêmico da paciente que normalizou após a retirada da medicação ${ }^{33}$.

A ocorrência de efeitos adversos impacta na adesão ao tratamento. Por isso, a avaliação do tratamento farmacológico e das comorbidades, a orientação sobre o uso correto dos medicamentos, horários, doses, interações e os possíveis efeitos adversos que o paciente pode apresentar, principalmente aqueles que utilizam vários medicamentos como frequentemente é encontrado em idosos, compõem todo o cuidado com o paciente ${ }^{10}$.

Em relação as interações medicamentosas com a rasagilina, foi encontrado que o uso concomitante de rasagilina com meperidina, tramadol, metadona $\mathrm{e}$ propoxifeno é desaconselhável pois há o risco de aumento dos níveis de serotonina resultando na síndrome serotoninérgica, caracterizada por agitação, confusão, rigidez, febre e mioclonia ${ }^{35}$. Ao utilizar inibidores da CYP1A2, associados a rasagilina, a dose máxima deve ser de 0,5mg/dia ${ }^{36}$ para evitar a ocorrência de reações adversas em razão da maior concentração do fármaco no sangue, consequente a interação medicamentosa do tipo farmacocinética de biotransformação ${ }^{37}$. São exemplos de inibidores da CYP1A2 os antimicrobianos ciprofloxacino, enoxacino, norfloxacino e ofloxacino, além da fluvoxamina que é um inibidor seletivo da recaptação de serotonina utilizado para tratar a depressão ${ }^{38}$.

Com tudo que foi apresentado, há evidências cientificas de que a rasagilina tem eficácia e segurança comprovada e 
que é possível o controle dos sintomas motores com o seu uso, especialmente nas associações com levodopa.

\section{CONCLUSÃO}

A partir dos resultados obtidos nesta revisão, concluise que a rasagilina é eficaz na redução dos sintomas motores da DP isolada ou associada a levodopa e é segura em razão a baixa ocorrência de reações adversas nos participantes dos estudos.

\section{REFERÊNCIAS}

1.Agência Nacional de Vigilância Sanitária. Consultas (endereço na Internet). 2015 (acessado em: 10/12/20). Disponível em: https://consultas.anvisa.gov.br/\#/medicamentos/253517548182013 $10 /$ ?substancia $=23672$ \&periodoPublicacaoInicial=2007-0101\&periodoPublicacaofinal $=2020-12-10$

2.Ministério da Saúde, Secretaria da Ciência, Tecnologia e Insumos Estratégicos. Portaria $N^{\circ} 27$, de 2 de agosto de 2017 (endereço na Internet). (acessado em: 10/12/20). Disponível em: https://www.in.gov.br/materia/-

Lasset publisher/Kujrw0TZC2Mb/content/id/19214639/do1-2017-0803-portaria-n-27-de-2-de-agosto-de-2017-19214561

3.Conitec. Mesilato de rasagilina como terapia adjuvante à levodopa para o tratamento de pacientes com doença de Parkinson com complicações motoras. (endereço na Internet). 2017 (acessado em: 17/08/20). Disponível em: http://conitec.gov.br/images/Relatorios/2017/Rasagilina_Parkinson_ 2 802017 FINAL.pdf

4.Brasil Ministério da Saúde. Portaria Conjunta $N^{\circ} 10$, de 31 de Outubro de 2017 (endereço na Internet). 2017 (acessado em: 14/05/20). Disponível em: http://www.saude.gov.br/images/pdf/2017/novembro/14/PortariaConjunta-PCDT-Doenca-de-Parkinson.pdf

5. Foppa AA. Qualificação do serviço farmacêutico clínico a partir dos dados de seguimento farmacoterapêutico a indivíduos com Doença de Parkinson (Tese). Florianópolis: Universidade Federal de Santa Catarina; 2014. https://repositorio.ufsc.br/bitstream/handle/123456789/123322/326 589. pdf? sequence $=1$ \&isAllowed $=y$ 
6. Teive HAG, Munhoz RP, Moro A, Moscovich M. Doença de Parkinson: Um guia para pacientes, familiares e cuidadores. São Paulo: Literatura Médica; 2017.

7.Silva FS, Pabis JVPC, De Alencar AG, Da Silva KB, Peternella FMN. Evolução da doença de Parkinson e comprometimento da qualidade de vida. Rev Neurocienc

2010;18:463-8.

https://doi.org/10.34024/rnc.2010.v18.8432

8. Finberg JPM. Pharmacology of Rasagiline, a New MAO-B Inhibitor Drug for the Treatment of Parkinson's Disease with Neuroprotective Potential. Rambam Maimonides Med J 2010;1:e0003. https://doi.org/10.5041/RMMJ.10003

9. Marchi KC, Chagas MHN, Tumas V, Miasso AI, Crippa JAS, Tirapelli $\mathrm{CR}$. Adesão à medicação em pacientes com doença de Parkinson atendidos em ambulatório especializado. Ciênc Saúde Col 2013; 18:855-62.

https://doi.org/10.1590/S1413-

81232013000300031

10.Foppa AA, Bevilacqua G, Pinto LH, Blatt CR. Atenção farmacêutica no contexto da estratégia de saúde da família. Rev Bras Cienc Farm 2008;44:727-37.

https://doi.org/10.1590/S1516-

93322008000400020

11.Brasil Mininstério da Saúde. Relação Nacional de Medicamentos Essenciais 2020 (endereço na Internet). (acessado em: 14/05/20). Disponível em: http://conitec.gov.br/images/Rename-2020-final.pdf

12.Secretaria de Estado da Saúde do Paraná, Coordenação de Assistência Farmacêutica do Paraná. Medicamentos do componentes básico, estratégico e especializado da relação nacional de medicamentos (RENAME) (endereço na Internet) 2019 (acessado em: 14/05/20). Disponível em: https://www.documentador.pr.gov.br/documentador/pub.do?action= d\&uuid=@gtf-escriba-sesa@a378dc58-b813-4017-bac7-

6299c176b6af\&emPg=true

13.Parkinson Study Group. A Randomized Placebo-Controlled Trial of Rasagiline in Levodopa-Treated Patients With Parkinson Disease and Motor Fluctuations: The PRESTO Study. Arch Neurol 2005;62:241-8. https://doi.org/10.1001/archneur.62.2.241

14.Parkinson Study Group. A Controlled Trial of Rasagiline in Early Parkinson Disease: The TEMPO Study. Arch Neurol 2002;59:1937-43. https://doi.org/10.1001/archneur.59.12.1937

15.Stocchi F, Rabey JM. Effect of rasagiline as adjunct therapy to levodopa on severity of OFF in Parkinson's disease: Rasagiline as adjunct to levodopa on severity of OFF. Eur J Neurol 2011;18:1373-8. https://doi.org/10.1111/j.1468-1331.2011.03512.x

16. Marsala SZ, Vitaliani R, Volpe D, Capozzoli F, Baroni L, Belgrado E, et al. Rapid onset of efficacy of rasagiline in early Parkinson's disease. Neurol Sci 2013;34:2007-13. https://doi.org/10.1007/s10072-0131437-2

17.Cereda E, Cilia R, Canesi M, Tesei S, Mariani CB, Zecchinelli AL, et al. Efficacy of rasagiline and selegiline in Parkinson's disease: a head- 
to-head 3-year retrospective case-control study. J Neurol 2017;264:1254-63. https://doi.org/10.1007/s00415-017-8523-y

18.Zhang L, Zhang Z, Chen Y, Qin X, Zhou H, Zhang C, et al. Efficacy and safety of rasagiline as an adjunct to levodopa treatment in Chinese patients with Parkinson's disease: a randomized, double-blind, parallel-controlled, multi-centre trial. Inter J Neuropsychopharmacol 2013;16:1529-37. https://doi.org/10.1017/S1461145713000175

19.Zhang Z, Shao M, Chen S, Liu C, Peng R, Li Y, et al. Adjunct rasagiline to treat Parkinson's disease with motor fluctuations: a randomized, double-blind study in China. Transl Neurodegener 2018;7:14. https://doi.org/10.1186/s40035-018-0119-7

20. Hattori N, Takeda A, Takeda S, Nishimura A, Kato M, Mochizuki H, et al. Efficacy and safety of adjunctive rasagiline in Japanese Parkinson's disease patients with wearing-off phenomena: A phase $2 / 3$, randomized, double-blind, placebo-controlled, multicenter study. Parkinson Related Disord 2018;53:21-7. https://doi.org/10.1016/j.parkreldis.2018.04.025

21. Hattori N, Takeda A, Takeda S, Nishimura A, Nakaya R, Mochizuki $\mathrm{H}$, et al. Long-term safety and efficacy of adjunctive rasagiline in levodopa-treated Japanese patients with Parkinson's disease. J Neural Transm 2019;126:289-97. https://doi.org/10.1007/s00702-0181962-5

22.Rascol O, Lozano A, Stern M, Poewe W. Milestones in Parkinson's disease therapeutics: Milestones in PD Therapeutics. Mov Disord 2011;26:1072-82. https://doi.org/10.1002/mds.23714

23.Shoulson I, Glaubiger GA, Chase TN. On-off response: Clinical and biochemical correlations during oral and intravenous levodopa administration in parkinsonian patients. Neurology 1975;25:1144. https://doi.org/10.1212/WNL.25.12.1144

24. Haddad F, Sawalha M, Khawaja Y, Najjar A, Karaman R. Dopamine and Levodopa Prodrugs for the Treatment of Parkinson's Disease. Molecules 2017;23:40. https://doi.org/10.3390/molecules23010040

25.Dezsi L, Vecsei L. Monoamine Oxidase B Inhibitors in Parkinson's Disease. CNS Neurol Disord Drug Targets 2017;16:425-39. https://doi.org/10.2174/1871527316666170124165222

26.Damiano AM, McGrath MM, Willian MK, Snyder CF, LeWitt PA, Reyes $\mathrm{PF}$, et al. Evaluation of a measurement strategy for Parkinson's disease: assessing patient health-related quality of life. Qual Life Res 2000;9:87-100. https://doi.org/10.1023/A:1008928321652

27. Hametner E, Seppi K, Poewe W. The clinical spectrum of levodopainduced motor complications. J Neurol 2010;257:268-75. https://doi.org/10.1007/s00415-010-5719-9

28. Witjas T, Kaphan E, Azulay JP, Blin O, Ceccaldi M, Pouget J, et al. Nonmotor fluctuations in Parkinson's disease: Frequent and disabling. Neurology 2002;59:408-13. https://doi.org/10.1212/WNL.59.3.408 29.Stocchi F, Jenner P, Obeso JA. When Do Levodopa Motor Fluctuations First Appear in Parkinson's Disease. Eur Neurol 2010;63:257-66. https://doi.org/10.1159/000300647 
30.Brunton LL. As bases farmacológicas da terapêutica de Goodman \& Gilman. $13^{\circ}$ ed. Porto Alegre: AMGH; 2019.

31. Follmer C, Bezerra Netto HJC. Fármacos multifuncionais: monoamina oxidase e \&\#945;-sinucleína como alvos terapêuticos na doença de Parkinson. Quím Nova 2013;36:306-13. https://doi.org/10.1590/S0100-40422013000200017

32.Schettino C, Dato C, Capaldo G, Sampaolo S, Di Iorio G, Melone MA. Rasagiline for sleep disorders in patients with Parkinson\&rsquo;s disease: a prospective observational study. Neuropsych Dis Treat 2016;12:2497-502. https://doi.org/10.2147/NDT.S116476

33.Ibrahim FAB, Rashid $F$, Hussain $A A B$, Alawadi $F$, Bashier $A$. Rasagiline-induced severe recurrent hypoglycemia in a young woman without diabetes: a case report. J Med Case Reports 2017;11:29. https://doi.org/10.1186/s13256-017-1202-x

34. Murad MH, Coto-Yglesias F, Wang AT, Sheidaee N, Mullan RJ, Elamin MB, et al. Drug-Induced Hypoglycemia: A Systematic Review. J Clin Endocrinol Metab 2009;94:741-5. https://doi.org/10.1210/jc.2008-1416

35.Teva. Anexo 1: Resumo das características do medicamento. (endereço na Internet). Agência Europeia de Medicamentos. (acessado em: 16/11/20). Disponível em:

https://www.ema.europa.eu/en/documents/product-

information/azilect-epar-product-information pt.pdf

36.Leegwater-Kim J. The role of rasagiline in the treatment of Parkinson's disease. Clin Intervent Aging 2010;149-56. https://doi.org/10.2147/CIA.S4145

37. Hoefler R. Interações Medicamentosas. Secretaria de Ciência, Tecnologia e Insumos Estratégicos/MS - FT (endereço na Internet). (acessado em: 16/11/20). Disponível em: http://www.toledo.pr.gov.br/intranet/ftn/docs/intMed.pdf

38.Golan DE, Tashjian Jr. AH, Armstrong EJ, Armstrong AW. Princípios de Farmacologia - A Base Fisiopatológica da Farmacologia. 3a. Rio de Janeiro: Guanabara Koogan; 2014. Disponível em: https://farmatecaunicatolica.files.wordpress.com/2017/12/a-basefisiopatolc3b3gica-da-farmacologia-golan-3c2aa-ed.pdf 